\title{
Article
}

\section{Facial emotion recognition and sleep in mentally disordered patients: A natural experiment in a high security hospital}

Chu, Simon, McNeill, Kimberley, Ireland, Jane L. and Qurashi, Inti Available at http://clok.uclan.ac.uk/12979/

Chu, Simon ORCID: 0000-0001-8921-4942, MCNeill, Kimberley, Ireland, Jane L. ORCID: 0000-0002-5117-5930 and Qurashi, Inti (2015) Facial emotion recognition and sleep in mentally disordered patients: A natural experiment in a high security hospital. Psychiatry Research, 230 (2). pp. 725-727. ISSN 01651781

It is advisable to refer to the publisher's version if you intend to cite from the work. http://dx.doi.org/10.1016/j.psychres.2015.10.005

For more information about UCLan's research in this area go to http://www.uclan.ac.uk/researchgroups/ and search for <name of research Group>.

For information about Research generally at UCLan please go to http://www.uclan.ac.uk/research/

All outputs in CLoK are protected by Intellectual Property Rights law, including Copyright law. Copyright, IPR and Moral Rights for the works on this site are retained by the individual authors and/or other copyright owners. Terms and conditions for use of this material are defined in the policies page.

\section{CLoK}

Central Lancashire online Knowledge www.clok.uclan.ac.uk

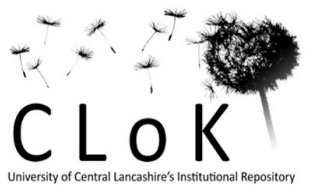


Facial emotion recognition and sleep in mentally disordered patients: A natural experiment in a high security hospital

Simon Chu*a,b Kimberley McNeill ${ }^{a, b}$, Jane L. Ireland ${ }^{a, b}, \&$ Inti Qurashi.

${ }^{a}$ Ashworth Research Centre, Ashworth Hospital, Mersey Care NHS Trust, Maghull L31 1HW, U.K.

${ }^{\mathrm{b} S}$ School of Psychology, University of Central Lancashire, Preston PR1 2HE, U.K.

*corresponding author, SChu@uclan.ac.uk

We investigated the relationship between a change in sleep quality and facial emotion recognition accuracy in a group of mentally-disordered inpatients at a secure forensic psychiatric unit. Patients whose sleep improved over time also showed improved facial emotion recognition while patients who showed no sleep improvement showed no change in emotion recognition.

Keywords: sleep; emotion-recognition

\section{Introduction}

Poor emotional processing is implicated in a broad range of mental disorders including personality disorders and schizophrenia. Individuals suffering from these disorders show pronounced deficits in identifying emotional expressions in the faces of others (e.g. Laroi et al., 2010; Daros et al., 2013). The ability to recognise emotions appropriately in others is crucial to competent social interaction and an inability to correctly identify emotions is thus likely to lead to social difficulties (Ihnen et al., 1998). An association between sleep and mental disorder has been noted (e.g. Sateia, 2009; Wulff et al., 2012), suggesting that that poor sleep hygiene (amount and quality of sleep achieved) may be a contributory factor to a deterioration in mental health. This is of particular importance to mental health inpatient units because research suggests that sleep quality deteriorates after admission, and that the decline in sleep quality is more marked for patients on mental health wards than on surgical or general wards (Doǧan et al., 2005). Several studies (e.g. Walker and van der Helm, 2009; van der Helm et al., 2010) have also shown that sleep deprivation may impair the recognition of emotional facial expressions. Thus, for patients suffering from mental disorders and poor sleep patterns, interventions to improve sleep quality may be of benefit to facial emotion recognition. 
Special circumstances arising in UK high security psychiatric hospitals presented an opportunity to conduct a natural experiment investigating sleep and emotion recognition. Ashworth Hospital, in the northwest of England, provides treatment for mentally disordered patients who pose a grave danger to the public and all patients at Ashworth Hospital have been legally detained under the Mental Health Act (2007). In 2011, the Department of Health directions concerning safety and security at the high security hospitals were revised to allow for the locked confinement of patients to their rooms overnight (between 21:15 and 07:15), a policy referred to here as Night Confinement (NC). Anecdotal reports suggested that before NC was implemented, the majority of patients tended to retire to bed late and wake late, or woke during the night and took naps during the daytime. An evaluation of the NC policy was undertaken (Chu et al., 2015) to assess the effects of the policy on patients and staff, and as a part of this evaluation, we examined the effect of NC on facial emotion recognition. With the implementation of NC, we hypothesised that an externally imposed structure would result in improved sleep quality and patients with improved sleep quality would be better able to correctly recognise facial emotions.

\section{Method}

Data were collected from six wards at Ashworth Hospital as part of a research programme examining the impact of the NC policy. There were 99 male inpatients resident on the six participating wards. The consultant forensic psychiatrist who was responsible for the treatment of each patient was approached for permission to approach their patients and clinicians granted permission to approach 84 of the patients (i.e. the remaining 15 patients were not stable enough to be approached by researchers to take part in a study). Of the 84 patients who were approached to participate in the study, 41 gave written informed consent to participation after the study had been explained to them (the other 43 patients declined to participate). Before the end of the study, 11 patients withdrew their consent leaving 30 patients (mean age: 39, age range: $23-60$ years; mean length of stay: 7 years, range: 9 months - 33 years) who completed the study in full. According to the clinical notes of completing patients, 23 patients had a main diagnosis of paranoid schizophrenia, three patients had a main diagnosis of schizoaffective disorder, three patients had a main diagnosis of dissocial personality disorder and one patient had a main diagnosis of emotionally unstable personality disorder. All diagnoses were made according to the International Statistical Classification of Diseases and Related Health Problems (ICD-10) by the patient's own consultant forensic psychiatrist at Ashworth Hospital; diagnoses were not independently confirmed by researchers. Aside from two of 
the three patients with a diagnosis of Dissocial Personality Disorder, all patients were prescribed antipsychotic medication (19 were taking clozapine, 3 were taking chlorpromazine, 2 were taking risperidone, with the remaining patients taking either flupenthixol, olanzapine, haloperidol, or quetapine). All patients continued with their prescribed medication and treatment programme as hospital inpatients during the course of the study and all medication doses remained stable over the course of the study.

Facial emotion recognition was measured by using a variant of the Ekman faces test (Young et al., 2010) using facial images from the Radboud Faces Database (Langner et al., 2010). 42 face images (21 male) were presented in colour (image size 2x4 inches), each on a single page of a booklet, expressing either happiness, sadness, surprise, anger, fear, disgust or a neutral expression (six of each expression). The names of all seven expressions were printed on the page and, for each face, the participant indicated which expression was being displayed. Participants were shown each expression until they made a response. Two versions of the test (versions $A$ and $B$ ) were produced which differed only in terms of the face images used and the presentation order of the emotions. The proportion of male to female faces, number of emotions and number of each emotional expression were kept the same in both versions of the test. Sleep quality was measured using the Pittsburgh Sleep Quality Index (PSQI; Buysse et al., 1988), a widely used self-report measure of sleep quality and quantity, measuring sleep latency, duration, efficiency, and a range of other indices of sleep quality. Higher scores indicate higher levels of sleep disturbance.

All participants completed the PSQI and the facial emotion recognition test between four and six weeks before NC was due to begin on their ward, and again 12 weeks after NC began. Half of the participants saw version A of the test pre-NC and version $B$ of the test post-NC, while the reverse was true for the other half of the participants. These data were collected as part of a broader research programme evaluating the impact of NC that was granted ethical approval by the Health Research Authority National Research Ethics Committee.

\section{Results}

Changes in sleep quality were computed for each participant by subtracting pre-confinement from post-confinement PSQI scores. Using the change scores, participants were divided into two groups; the improved sleep group (negative change in PSQI) and the non-improved sleep group (no change 
or positive change in PSQI). 16 participants (13 with a diagnosis of paranoid schizophrenia, one with a diagnosis of schizoaffective disorder, one with a diagnosis of dissocial personality disorder and one with a diagnosis of emotionally unstable personality disorder) reported improved sleep (mean change: -3.9 , SD: 2.5 , range: -1 to -9 ) and 14 participants (10 with a diagnosis of paranoid schizophrenia, two with a diagnosis of dissocial personality disorder, and two with a diagnosis of schizoaffective disorder) reported no improvement (mean change: 2.1, SD: 2.0, range: 0 to 6 ). The facial emotion recognition rates for the two groups for each of the seven expressions are shown in Table 1.

Figures in Table 1 suggest that participants recognised happiness and surprise quite well but were less able to recognise fear and anger, and in terms of total emotion recognition, the improved sleep group showed an increase in emotion recognition ability (pre: 30.19, post: 32.25 ) but the nonimproved sleep group did not (pre: 31.93, post: 31.50). Emotion recognition data were entered into a three-way (sleep group $x$ time $x$ emotion) mixed analysis-of-variance with time and emotion as repeated factors. Mauchly's test indicated that the assumption of sphericity had been violated for the main effect of emotion, $\chi^{2}(20)=48.3, p<.001$, and therefore degrees of freedom were corrected using a Greenhouse-Geisser estimate of sphericity $(\varepsilon=.66)$. All effects are reported as significant at $p<.05$. There was a significant main effect of emotion, $F(3.94,110.26)=48.54$, and contrasts revealed that in comparison to the neutral expression, participants were significantly less able to recognise expressions of anger, $F(1,28)=14.59$, and fear, $F(1,28)=86.68$, whereas they were significantly more able to recognise expressions of happiness, $F(1,28)=25.78$, and surprise, $F(1,28)=$ 7.90. There was also a significant interaction between sleep group and time, $F(1,28)=4.56$, and a simple effects analysis revealed that the improved sleep group showed a significant increase in emotion recognition from pre- to post confinement, $F(1,28)=6.70$, whilst the non-improved sleep group did not, $F(1,28)=0.25$. All other effects and interactions were not significant.

\section{Discussion}

Specific mental health disorders and reduced sleep quality are both known to contribute to impaired facial emotion recognition. The ability to recognise facial emotions is an important aspect of social cognition, is a core feature in developing social relationships and, for individuals with a mental disorder, may be a crucial part of recovery (Penn et al., 1997). In this study we have shown that patients whose sleep hygiene improved also experienced an improvement in their ability to correctly 
recognise facial emotions; conversely patients who experienced no improvement in sleep hygiene showed no change in emotion recognition.

Neuroimaging studies suggest that neural systems that suffer from sleep deprivation have a substantially amplified amygdala response to negative stimuli coupled with a breakdown in prefrontal control; that is, a dysregulation in autonomic affective physiology (Yoo et al., 2007; Killgore, 2013). The deficits in emotion processing that are observed in individuals with schizophrenia have also been linked directly with abnormal amygdala activation, and in particular, when negative emotion is involved (Takahashi et al., 2004). Few studies have directly examined the effects of medication that has been implicated in amygdala activation but a small number of compounds, including citalopram (Del-Ben et al., 2004), sulpride (Takahashi et al., 2005), levodopa (Delaveau et al., 2005) and lorazepam (Paulus et al., 2005) have shown decreased amygdala activation compared to placebo during the processing of emotional stimuli. However the evidence concerning the effect of antipsychotic medication on emotion perception abilities is mixed, and Pinkham, Gur and Gur (2007) have concluded that antipsychotics are largely ineffective in improving affect perception. In the present sample, the vast majority of participants were taking one of seven different antipsychotic medications, but a large scale clinical trial of 1493 patients with a diagnosis of schizophrenia comparing the effects of five different antipsychotics (Penn et al., 2009) showed that changes in emotion perception were equivalent across all five compounds (olanzapine, perphenazine, quetiapine, risperidone and ziprasidone). Thus the effects on emotion perception shown in the present data are unlikely to have been affected by differences in the medication status of patients.

It is likely that the effects shown here may be more pronounced for facial emotions of lower intensity. The face images used here expressed strong and unambiguous emotional expressions but previous work suggests that sleep exerts a greater effect on the recognition of less intense expressions (van der Helm et al., 2010). More subtle emotional expressions are likely to require a more sophisticated level of emotion perception, and it may be that the effect of good quality sleep is most evident in the facilitation of such affective perceptual processes.

However, it is unclear how sleep differentially affects the recognition of different types of emotion and future studies should try to differentiate the effect of sleep on the recognition of different expressions. The present data were collected as part of a broader study examining the impact of the night confinement policy and was therefore limited in the time that could be devoted to facial 
emotion recognition. Given the relatively small sample, replication of the results with a greater number of participants would place our conclusions on a more reliable footing and a more focused investigation of the recognition of different emotional expressions would make a valuable contribution. Nevertheless, these preliminary data contribute to the growing consensus in favour of greater efforts to improve sleep quality in inpatient mental health care (e.g. Wilson and Argyropoulos, 2012). We did not examine the possible corollaries of improved emotion perception (such as peer relationship quality) and it is possible that other improvements in patient presentation as a result of improvements in sleep hygiene may be evident. Further work should examine this possibility. 


\section{References}

Buysse, D.J., Reynolds, C.F., Monk, T.H., Berman, S.R., Kupfer, D.J., 1989. The Pittsburgh Sleep Quality Index - a new instrument for psychiatric practice and research. Psychiatry Research 28 (2), 193-213.

Chu, S., McNeill, K., Wright, K.M., Hague, A., Wilkins, T., 2015. The impact of a night confinement policy on patients in a UK high secure inpatient mental health service. Journal of Forensic Practice 17 (1), 21-30.

Daros, A.R., Zakzanis, K.K., Ruocco, A.C., 2013. Facial emotion recognition in borderline personality disorder. Psychological Medicine 43 (9), 1953-1963.

Delaveau, P., Salgado-Pineda, P., Wicker, B., Micallef-Roll, J., Blin, O. 2005. Effect of levodopa on healthy volunteers' facial emotion perception - an fMRI study. Clinical Neuropharmacology 28 (6), 255-261.

Del-Ben, C.M., Deakin, J.F.W., McKie, S., Delvai, N.A., Williams, S.R., Elliott, R., Dolan, M., Anderson. I.M. 2005. The effect of citalopram pretreatment on neuronal responses to neuropsychological tasks in normal volunteers: An fMRI study. Neuropsychopharmacology 30 (9), 1724-1734.

Doğan, O., Ertekin, S., Doǧan, S., 2005. Sleep quality in hospitalized patients. Journal of Clinical Nursing 14 (1), 107-113.

Ihnen, G.H., Penn, D.L., Corrigan, P.W., Martin, J. (1998). Social perception and social skill in schizophrenia. Psychiatry Research 80 (3), 275-286.

Killgore, W.D.S., 2013. Self-Reported Sleep Correlates with Prefrontal-Amygdala Functional Connectivity and Emotional Functioning. Sleep 36 (11), 1597-1608.

Langner, O., Dotsch, R., Bijlstra, G., Wigboldus, D.H.J., Hawk, S.T., van Knippenberg, A., 2010. Presentation and validation of the Radboud Faces Database. Cognition \& Emotion 24 (8), 1377-1388.

Laroi, F., Fonteneau, B., Mourad, H., Raballo, A., 2010. Basic emotion recognition and psychopathology in schizophrenia. Journal of Nervous and Mental Disease 198 (1), 79-81.

Paulus, M.P., Feinstein, J.S., Castillo, G., Simmons, A.N., Stein, M.B. 2005. Dose-dependent decrease of activation in bilateral amygdala and insula by lorazepam during emotion processing. Archives of General Psychiatry 62 (3), 282-288.

Penn, D.L., Corrigan, P.W., Bentall, R.P., Racenstein, J.M., Newman, L., 1997. Social cognition in schizophrenia. Psychological Bulletin 121 (1), 114-132.

Penn D.L., Keefe R.S.E., Davis S.M., Meyer P.S., Perkins D.O., Losardo D. and Lieberman J.A., The effects of antipsychotic medications on emotion perception in patients with chronic schizophrenia in the CATIE trial, Schizophrenia Res. 115(1), 2009, 17-23.

Pinkham A.E., Gur R.E. and Gur, R.C., Affect recognition deficits in schizophrenia: neural substrates and psychopharmacological implications, Expert Review of Neurotherapeutics 7(7), 2007, 807-16.

Sateia, M.J., 2009. Update on sleep and psychiatric disorders. Chest 135 (5), 1370-1379. 
Takahashi, H., Koeda, M., Oda, K., Matsuda, T., Matsushima, E., Matsuura, M., Asai, K., Okubo, Y., 2004. An fMRI study of differential neural response to affective pictures in schizophrenia. Neuroimage 22 (3), 1247-1254.

Takahashi, H., Yahata, N., Koeda, M., Takano, A., Asai, K., Suhara, T., Okubo, Y. 2005. Effects of dopaminergic and serotonergic manipulation on emotional processing: A pharmacological fMRI study. Neuroimage 27 (4), 991-1001.

van der Helm, E., Gujar, N., Walker, M. P., 2010. Sleep deprivation impairs the accurate recognition of human emotions. Sleep 33 (3), 335-342.

Walker, M. P., van der Helm, E., 2009. Overnight therapy? The role of sleep in emotional brain processing. Psychological Bulletin 135 (5), 731-748.

Wilson, S. \& Argyropoulos, S., 2012. Sleep in schizophrenia: time for closer attention. British Journal of Psychiatry 200 (4), 273-274.

Wulff, K., Dijk, D., Middleton, B., Foster, R.G., Joyce, E.M., 2012. Sleep and circadian rhythm disruption in schizophrenia. British Journal of Psychiatry 200 (4), 308-316.

Yoo, S.S., Gujar, N., Hu, P., Jolesz, F.A., Walker, M.P., 2007. The human emotional brain without sleep - a prefrontal amygdala disconnect. Current Biology 17 (20), R877-8.

Young, A.W., Perrett, D.I., Calder, A.J., Sprengelmeyer, R., Ekman, P., 2010. Facial Expressions of Emotion: Stimuli and Tests (FEEST). Thames Valley Test Co., Bury St. Edmunds. 
Table 1. Mean number of facial emotion expressions of each type correctly recognised (standard deviation) by patients whose sleep improved after night confinement $(n=16)$ and patients whose sleep did not improve $(n=14)$.

\begin{tabular}{lccccc} 
& \multicolumn{2}{c}{ Improved } & & \multicolumn{2}{c}{ Non-improved } \\
\cline { 2 - 3 } \cline { 6 - 6 } Expression & Pre & Post & & Pre & Post \\
\hline Angry & $3.69(1.78)$ & $3.19(1.68)$ & & $3.43(1.79)$ & $3.50(1.51)$ \\
Disgust & $4.13(1.59)$ & $5.00(1.03)$ & & $4.57(1.55)$ & $4.36(1.98)$ \\
Afraid & $1.69(1.54)$ & $1.81(1.52)$ & & $2.50(1.79)$ & $2.29(1.94)$ \\
Happy & $5.81(0.75)$ & $6.00(0.00)$ & & $5.93(0.27)$ & $6.00(0.00)$ \\
Surprise & $5.38(1.02)$ & $5.50(0.73)$ & & $5.64(0.50)$ & $5.36(0.93)$ \\
Sad & $4.81(1.11)$ & $5.56(0.81)$ & & $5.29(0.99)$ & $5.29(1.20)$ \\
Neutral & $4.69(1.20)$ & $5.19(0.91)$ & & $4.57(1.50)$ & $4.71(1.59)$ \\
\hline Total & $30.19(4.10)$ & $32.25(2.67)$ & & $31.93(3.63)$ & $31.50(5.43)$
\end{tabular}

